\title{
Accents in equitone sequences
}

\author{
DIRK-JAN POVEL and HANS OKKERMAN \\ University of Nijmegen, Nijmegen, The Netherlands
}

\begin{abstract}
Equitone sequences are defined as sequences of tones that are identical in all respects: frequency, spectral composition, intensity, and duration. The only parameter varied in these sequences is the time-interval between tones. In such sequences, clear accents are perceived. This paper describes accent perception in equitone sequences containing two alternating intervals; such sequences are perceived as consisting of repeating groups of two tones. An accent is heard on the first tone of a group if the difference between the intervals is about $5 \%$ to $10 \%$. If the difference is made bigger, the accent is heard on the last tone of the group; this latter accent is considerably stronger than the accent previously heard on the first tone. In a number of experiments, the conditions under which the two types of accents occur were investigated. From these experiments, it was tentatively concluded that the accent on the last tone is heard because that tone, since it is followed by a longer interval, can be processed more completely. This "intervalproduced" accent indeed occurs only if the between-group interval is considerably longer than the within-group interval and if the latter does not exceed a duration of about $250 \mathrm{msec}$. The effect is slightly dependent on tone duration. The interval-produced accent can be balanced if the nonaccented tone is increased by about $4 \mathrm{~dB}$ in intensity. This shows that the effect is quite robust. The specific type of accentuation reported here might explain some rhythmical phenomena, examples of which are given.
\end{abstract}

It is generally agreed that accents have an important influence on the perception of tone sequences. Accentuating a tone in a sequence can be accomplished by making that tone louder or longer in duration than its neighboring tones. These accents, respectively called dynamic and temporal accents (Cooper \& Meyer, 1971), were studied extensively by Woodrow (1909), and melodic accents due to specific features in the melodic line have recently been studied by Thomassen (Note 1).

While studying equitone sequences (sequences generated from tones identical in all respects), it was found that distinct accents were perceived in such sequences also. Since these accents may play an important role in the coding of the temporal aspects of equitone sequences (Povel, 1981), they deserve a more careful study.

Unlike the types of accentuation mentioned above, the accents in equitone sequences are not perceived because of differences between characteristics of the elements (tones) themselves. We conjectured, therefore, that the accents are not caused by local determinants but, rather, are the result of the perceived structure of the sequence, that is, that they are caused by the perceived grouping of the tones in the sequence. This led to the expectation that accents would be heard on the first tone of perceived groups

\footnotetext{
The authors wish to thank Herbert Pick for reading and commenting upon an earlier draft of this paper. Requests for reprints should be sent to Dirk-Jan Povel, Department of Experimental Psychology, University of Nijmegen, Postbus 9104, 6500 HE Nijmegen, The Netherlands.
}

as it is in the "subjective rhythm" phenomenon that can arise when listening to isochronic sequences (equitone sequences with identical intervals). In hearing such sequences, subjects have reported the sequences as consisting of groups of two, three, or four tones, with an accent on the first tone of each group (Fraisse, 1956; Harrell, 1937; Vos, 1977).

In an initial pilot study, we determined that when subjects are first presented with an isochronic sequence, generated from 50 -msec tones and 50 -msec intervals, and then every second interval is gradually increased, the subjects hear groups of two tones, the longer interval separating the groups as soon as the longer interval exceeds the shorter by $5 \%$ to $10 \%$. If asked, subjects will report an accent on the first tone of the group. If the second interval is further increased, the perceived grouping remains unchanged, but the accent seems to move to the second tone of the group. When the difference between the two intervals exceeds a certain value, subjects consistently report hearing an accent on the second tone. The accent remains on the last tone with a further increase of the longer interval. The accent on the second tone appears to be stronger than the accent previously heard on the first tone. If this same experiment is repeated now with every third interval, rather than every second interval, gradually increasing, subjects report hearing groups of three tones and an accent on the first tone of each group as soon as the increased interval is again about $5 \%$ to $10 \%$ longer than the others. With a further increase of the third interval, in addition to the accent on the first tone a stronger accent is reported on the last tone of the group. 
From this first exploration, it seemed unlikely that the perceived accents in equitone sequences were a consequence of the perceived grouping alone. In the case of the last tone of the group, especially, the accent seems to be determined by another cause, namely, the relative length of the interval following the tone. Moreover, this latter accent is much more prominent than the accent perceived on the first tone of a group in the less differentiated sequences: it dominates the accent on the first tone in the two-tone case and is much stronger than the accent on the first tone in the three-tone case. This type of "intervalproduced" accent has not previously been reported in the literature, although there are a few studies of related phenomena.

Possibly related to the interval-produced accent are the so-called "time error" studies. Koehler (1923) examined loudness judgments of two short clicks separated by intervals varying from 1.5 to $6 \mathrm{sec}$ and reported that for intervals of up to $3 \mathrm{sec}$ the second click was judged softer, whereas for longer intervals the second click was judged louder. Similar data have been published by Needham (1934) and Postman (1946), who also used interstimulus intervals of $1 \mathrm{sec}$ up to several seconds and tones with a duration of at least $200 \mathrm{msec}$; these authors found that the second sound was judged louder. A study employing interstimulus intervals closer to our pilot study was conducted by Buytendijk and Meesters (1942). Their subjects were presented with pairs of clicks and were requested to adjust the intensity of the second click until it matched the first one in loudness. Buytendijk and Meesters reported that their subjects made the second click progressively weaker as the interval between the two clicks was decreased from 200 to 10 msec. They explained their finding by assuming "integration" of the two clicks: residual sensation from the first click is added to the sensation of the second. Elmasian and Galambos (1975) have challenged this explanation. They argued that the first tone is not louder because of the presence of the first, but rather that the first is weakened by the presence of the second. Vos (1977) presented his subjects with periodic sequences and asked them to indicate whether they had heard an iambic or a trochaic pattern. Two of his stimuli were equitone sequences with tone durations of 80 and $320 \mathrm{msec}$, respectively. In both sequences, interval durations of 80 and $320 \mathrm{msec}$ were alternated. Eighty-five percent of the responses were iambic for the sequence with the short tone duration and $47.5 \%$ were iambic for the sequence with the 320-msec tones.

Related to the latter investigation are the "loudness enhancement" studies. Loudness enhancement refers to the fact that under certain conditions the loudness of a tone is enhanced if it is preceded by another tone. All studies investigating this phenomenon, however, have shown that no loudness enhance- ment is found if the two tones are of the same frequency (Zwislocki, Ketkar, Cannon, \& Nodar, 1974) or if the tones are of the same intensity (Elmasian \& Galambos, 1975; Zwislocki \& Sokolich, 1974). Thus, loudness enhancement can be readily discarded as an explanation for the findings of our pilot study since we used only sequences containing identical tones.

The interval-produced accent that was found in our pilot study might also be related to a form of masking known as "partial masking," in which the loudness of an auditory element is decreased by the presence of another auditory element. Most of the research on masking has used a total masking paradigm in which the detection of a stimulus is examined as a function of a preceding or a following sound, respectively called forward and backward masking. The available research is inconclusive as to the relative strengths of forward and backward masking. Some authors claim that forward masking is the stronger (Elliot, 1962a; Wilson \& Carhart, 1971), while others claim that backward masking is stronger (Elliot, 1962b; Samoilova, 1959). Samoilova used tones rather than clicks, so these findings may be more relevant to our study. Unfortunately, however, the test tone and masker had different frequencies and completely different durations (20 and $300 \mathrm{msec}$ ). The differences actually found between forward and backward masking are practically quite small. All studies agree that masking activity does not last much longer than 100 to $150 \mathrm{msec}$.

Another possible explanation is related to the concept of echoic memory, or preperceptual auditory store, which states that the information in the stimulus is kept in a store for a short amount of time after termination of the stimulus, where it is available for processing (Massaro, 1972; Neisser, 1967). Massaro (1970) demonstrated that recognition of the pitch of a tone was disturbed if another tone was presented within a period of about $250 \mathrm{msec}$ after presentation of the stimulus. The implication was that the processing of an attribute such as the pitch of a tone takes about $250 \mathrm{msec}$ and that this processing is interrupted if, within that interval, new information is fed into echoic store. Although the attribute under consideration here is clearly different from pitch, it still seems reasonable to assume that if the processing of a tone is interrupted its strength might be underestimated.

The experiments reported here were designed to establish the limits and the strengths of the intervalproduced accent and to decide upon an adequate explanation of the phenomenon.

\section{EXPERIMENT 1}

This experiment was designed to determine the conditions under which the interval-produced accent in equitone sequences occurs. It was decided to study 
accent perception in sequences consisting of repeated two-tone groups, rather than three-tone groups, first, because two-tone groups are the simpler types, and second, because in three-tone sequences no forced-choice responding could be used since two accents may be heard in every group. It was decided to use a value of $50 \mathrm{msec}$ for the duration of the tones and a value of $50 \mathrm{msec}$ for the smallest occurring interval in order to have durations in the range of musical sequences [the smallest note value (interonset interval) occurring in music is approximately $100 \mathrm{msec}]$.

\section{Method}

The stimuli used in the experiment consisted of sequences of $1,000-\mathrm{Hz}$ sine-wave tones, with a duration of $50 \mathrm{msec}$ and rise and fall times of 7.5 msec presented at a level of $50 \mathrm{~dB}$ SPL. In all sequences, two different interstimulus intervals (i1 and i2) were alternated repeatedly. All sequences started with i1, always the smaller of the two intervals. In 10 of the 19 stimulus sequences used, il was fixed at 50 misec and $\mathrm{i} 2$ was varied from 70 to $340 \mathrm{msec}$ in 30 -msec steps. In the $\theta$ other sequences, i2 was fixed at $340 \mathrm{msec}$ and il was varied from 80 to $320 \mathrm{msec}$, also in 30 -msec steps. The temporal structure of the stimuli used are presented in Table 1 and displayed in Figure 1. The sequences were presented through headphones in a random order. The subjects listened to each sequence as long as they liked and indicated, by using a response button, whether they perceived an accent on the first or second tone of the group. Both stimulus generation and response collection were controlled by a PDP-11/34 computer. Before the actual experiment, the subjects practiced with 20 representative sequences. Three subjects, two male and one female, all graduate students in psychology, participated in the experiment. Each subject judged each stimulus 25 times, in a total of five sessions during a 2 -week period.

\section{Results}

The subjects heard all sequences as repeated groups of two tones, separated by the longer interval, i2. Therefore, which tone was accented could be

Table 1

Temporal Characteristics of the Stimuli Used in Experiment 1

\begin{tabular}{cccccc}
\hline $\begin{array}{c}\text { Stimulus } \\
\text { Hour }\end{array}$ & $\begin{array}{c}\text { Tone } \\
\text { A }\end{array}$ & $\begin{array}{c}\text { Interval } \\
1\end{array}$ & $\begin{array}{c}\text { Tone } \\
\text { B }\end{array}$ & $\begin{array}{c}\text { Interval } \\
2\end{array}$ & $\Delta t(\mathbf{i} 2-\mathbf{i} 1)$ \\
\hline 1 & 50 & 50 & 50 & 70 & 20 \\
2 & 50 & 50 & 50 & 100 & 50 \\
3 & 50 & 50 & 50 & 130 & 80 \\
4 & 50 & 50 & 50 & 160 & 110 \\
5 & 50 & 50 & 50 & 190 & 140 \\
6 & 50 & 50 & 50 & 220 & 170 \\
7 & 50 & 50 & 50 & 250 & 200 \\
8 & 50 & 50 & 50 & 280 & 230 \\
9 & 50 & 50 & 50 & 310 & 260 \\
10 & 50 & 50 & 50 & 340 & 290 \\
11 & 50 & 80 & 50 & 340 & 260 \\
12 & 50 & 110 & 50 & 340 & 230 \\
13 & 50 & 140 & 50 & 340 & 200 \\
14 & 50 & 170 & 50 & 340 & 170 \\
15 & 50 & 200 & 50 & 340 & 140 \\
16 & 50 & 230 & 50 & 340 & 110 \\
17 & 50 & 260 & 50 & 340 & 80 \\
18 & 50 & 290 & 50 & 340 & 50 \\
19 & 50 & 320 & 50 & 340 & 20 \\
\hline
\end{tabular}

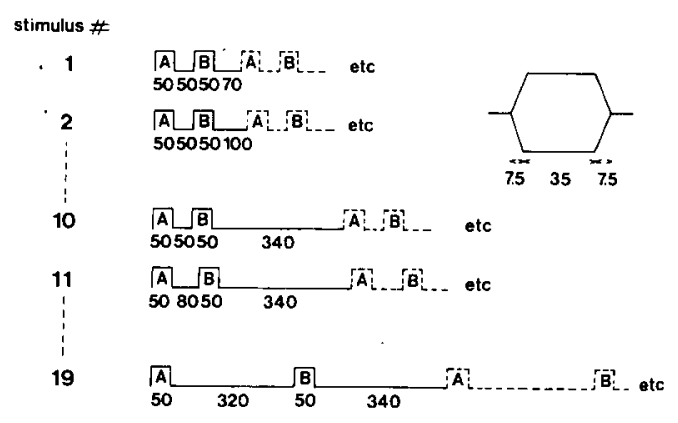

Figure 1. Schematic display of the stimull used in Experiment 1. The tones are 1,000-Hz sine waves at $50 \mathrm{~dB}$ SPL; durntion is 50 msec, Including 7.5-msec rise and fall time. Insert: tone envelope.

reliably indicated by referring to the position of the accented tone in the group. The location of the accented tone is indicated in Figure 2. The left half of the figure shows the 10 stimuli with il fixed at. $50 \mathrm{msec}$, whereas the part at the right side of the dashed vertical shows the 9 stimuli with i2 fixed at $340 \mathrm{msec}$. First consider the il-fixed sequences. As long as i 2 is relatively short, subjects indicate hearing an accent on the first tone. Then, as $\mathrm{i} 2$ gradually increases, more and more often the accent is heard on the last tone of the group, until an asymptotic value is reached. The $\mathrm{i} 2$ duration at which the asymptotic values are reached are quite different for the three subjects: approximately 130,220 , and $310 \mathrm{msec}$. One subject (Subject 1) shows a slight tendency to hear less accent on the last tone with more extreme values of i2. The right side of the figure shows that if i 2 is fixed at $340 \mathrm{msec}$ and il is gradually increased, all subjects first hear the accent on the last tone, which moves to the first tone with increasing values of il. Again, we find considerable differences among the subjects: the accent starts to move away from their asymptotic values at values, for $\mathrm{il}$, of approximately 50,140 , and $230 \mathrm{msec}$ for the three subjects, in the same order.

\section{Discussion}

If there is only a small difference between the two intervals used, subjects report an accent on the first tone of the group. This is apparently the base response for temporally rather undifferentiated sequences. If the difference between intervals is increased, at some point a tendency is shown to hear the accent on the last tone. This finding is in agreement with the results of Buytendijk and Meesters (1942) and with the result of Vos (1977) for the 80msec equitone sequence mentioned above. From the reactions to the il-fixed sequences (left side of Figure 2), it appears that subjects report an accent on the second tone of the group if the between-group interval (i2) is on the average at least $220 \mathrm{msec}(\Delta \mathrm{t}=$ $170 \mathrm{msec}$ ). The responses to the i2-fixed stimuli show 
that if il is, on the average, larger than $140 \mathrm{msec}$ $(\Delta t=200 \mathrm{msec})$, the tendency to hear the accent on the last tone starts to disappear. These average values might suggest that the absolute difference between the two interval determines the accent: If the difference exceeds approximately $180 \mathrm{msec}$, a tendency to hear an accent on the second tone of each group will be apparent.

This suggestion would be correct if the three curves were symmetrically shaped around the dotted vertical at $\Delta \mathrm{t}$ of $290 \mathrm{mec}$. This, however, is clearly not the case: Subject 1 , for instance, reports $100 \%$ accent on the second tone of each group for i1 and i2 of, respectively, 50 and $130 \mathrm{msec}(\Delta t=80 \mathrm{msec})$, but this same subject reports only $25 \%$ accent on the second tone for the same $\Delta t$ but values for i1 and i2 of, respectively, 260 and $340 \mathrm{msec}$. The typical asymmetries in the curves of the three subjects (an early rise implying a slow fall, and vice versa) can be explained by assuming different decays of the internal persistence of the stimulus in echoic store. Plomp (1964) reports a difference of about $100 \mathrm{msec}$ between the asymptotic values of the decay curves of his two subjects (200 vs. $300 \mathrm{msec})$. If we assume that the perceived strength of a tone depends on the duration of the stimulus plus the uninterrupted part of the persistence, we could explain the asymmetry in the curves by assuming different decays. Consider a person with a short decay. In the first part of the experiment (the il-fixed sequences), as i2 increases the maximum difference between the strength of the two tones will soon be reached. In the second part of the experiment, in which il is increased, the point at which the strength of the two tones are equal will soon be reached. Such a person will produce the curve of Subject 1. A person with a long decay will produce the curve of Subject 3. Figure 3 illustrates the reasoning presented here. Of course, this model does not explain the perceived accents on the first

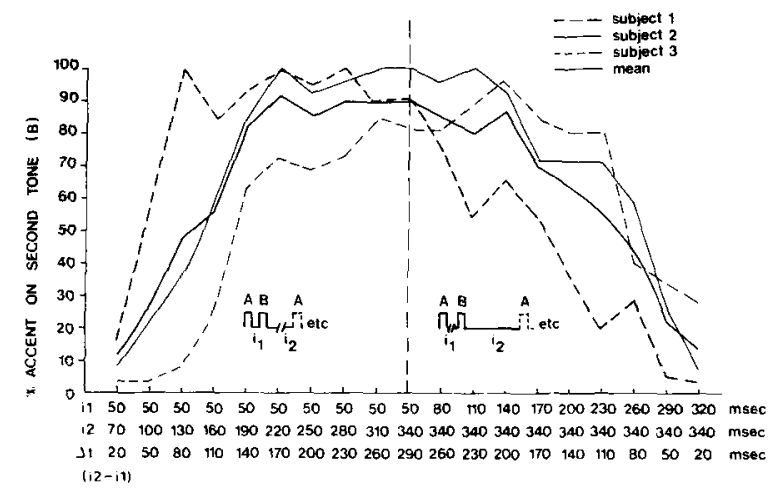

Figure 2. Percentage of perceived accent on lust tone of group in sequences consisting of repeated groups of two tones, as a function of the duration of the intervals il and i2. Tone duration = 50 msec; durations of intervals are indicated in the figure. Inserts: one perod of sequence.

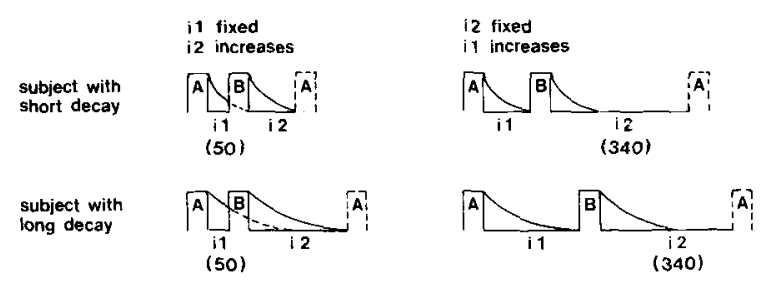

Figure 3. Tone strength and decay, for two hypothetical subjects with, respectively, a short and a long decay. The left-hand side shows the configuration at which the maximum difference between the strengths of tone $A$ and tone $B$ has been reached by lncreasing 12 (11 = $50 \mathrm{msec})$. The right-hand side shows the configuration at which tones $A$ and $B$ are perceived to be equally strong by increasing 11 ( $12=340 \mathrm{msec})$.

tone in some of the sequences. Experiment 2 studied this phenomenon.

It should be realized that the observed asymmetries can also be explained by assuming that the processing times needed by the subjects differ. A fast processor would produce the curve of Subject 1 , a slow processor the curve of Subject 3. Massaro (1970), does not find different processing times for his three subjects, but his study does not include many measuring points. If the interval-produced accent is the result of the integration of the energy of the stimulus plus its (uninterrupted) decay, it should be realized that the energy contributed by the decay or persistence is very small: According to Plomp (1964), the persistence of a 45 - $\mathrm{dB}$ tone has decayed over $35 \mathrm{~dB}$ after $100 \mathrm{msec}$; a comparable value was reported by Samoilova (1959) in a forward masking study. Experiment 3 was especially conducted to decide between the two explanations mentioned here. First we will describe Experiment 2, which investigates the cause of the reported accent on the first tone.

\section{EXPERIMENT 2}

This experiment was done to determine whether the accent on the first tone, reported in those sequences with only small differences between the two intervals, was the result of the perceived grouping, or whether it was partly or completely determined by an orienting response to that tone. The orienting response would give rise to an accent on the first tone of the sequence and, by induction, on all other first tones of the periodically recurring groups. Note that the very first tone is preceded by a relatively long empty interval. If the accent on the first tone is completely determined by this contrast and the resulting orienting response, we would expect the accent on the first tone to disappear if, at a short interval before each sequence, a long tone were presented.

\section{Method}

Some of the stimuli used in Experiment 1 were also used in this experiment, namely those for which an orienting response could 


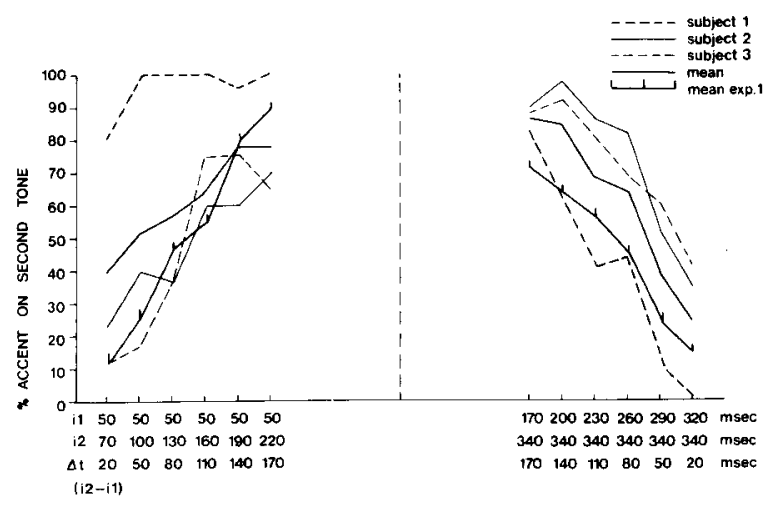

Figure 4. Percentage of perceived accent on last tone of group in sequences consisting of repeated groups of two tones, as a function of the duration of the intervals 11 and 12 . Tone duration $=50 \mathrm{msec}$; durations of intervals are indicated in the figure. Each sequence is preceded by a $500-m s e c$ tone $(1,000-\mathrm{Hz}$ sine wave, $50 \mathrm{~dB})$ at an Interval equal to 12.

be expected to play a role in the perceived accent. These are the stimuli with relatively small $\Delta t$ values. Hence, all stimuli with a $\Delta t$ value of $170 \mathrm{msec}$ or less were included. Each sequence was preceded by a $1,000-\mathrm{Hz}$ tone with the same intensity as the other tones in the sequence and a duration of $500 \mathrm{msec}$ and rise and fall times of $7.5 \mathrm{msec}$. The interval between the long initial tone and the sequence was equal to i2, the between-group interval. Stimulus presentation and response collection were identical to those used in Experiment 1. The subjects were the same as those used in Experiment 1.

\section{Results and Discussion}

The results presented in Figure 4 indicate adding the 500-msec tone to the sequence did have an effect. On the average, the percentage of accents on the final tone is slightly higher. The difference from the curves obtained in Experiment 1, which did not have a preceding tone, is rather small, however. Therefore, it seems unwarranted to conclude that the initial accent can be accounted for completely by an orienting reaction. Apparently, besides this factor, there is the influence of what has been called a structural accent, that is, the tendency to hear an accent on the first tone of a perceived group.

\section{EXPERIMENT 3}

Experiment 3 was designed to test the energyintegration hypothesis mentioned in the discussion of Experiment 1. According to this hypothesis, the difference in strength between the two tones is determined by the difference in overlap of the decay parts of the two tones. Since the overlap involves only the tails of the persistence, the effect must be quite small. The effect should, however, become even smaller, or vanish, if the tones are made longer in duration. The longer the tones, the less the influence of the difference in overlap of the decays. Therefore, in this experiment tones of $150 \mathrm{msec}$ duration were combined with the same interval pairs as those used in Experiment 1 (see Table 1). A tone duration of $150 \mathrm{msec}$ combined with the longest interval used $(340 \mathrm{msec})$ yields an interonset interval of about $.5 \mathrm{sec}$, which belongs to the longer note values occurring in music. Note that the present hypothesis assumes that energy is integrated and contributes to loudness or strength over a period of more than 150 msec. All authors studying temporal summation have reported, however, that the critical value above which loudness no longer increases with duration is a maximal 150 msec (Boone, 1973; Reichardt \& Niese, 1970; Zwislocki, 1969). This is another reason why no accents on the second tone are to be expected in this experiment on the basis of the energy-integration hypothesis.

\section{Method}

The stimuli used differed from those of Experiment 1 only in the duration of the tones, which was now $150 \mathrm{msec}$. The temporal characteristics of the intervals were identical. The same three subjects participated, and the same methods of stimulus presentation and response collection were used.

\section{Results and Discussion}

The accents perceived in the sequences used are displayed in Figure 5. The overall impression is that, for these stimuli as well, an accent on the last tone of the groups is heard if the difference between il and i2 exceeds some value. This value again differs for the three subjects. It is clear that the intervalproduced accents cannot be the result solely of energy integration, although lengthening the tones does have a slight effect. Essentially the same accent perception is found as in Experiment 1. If the results of Experiments 1 and 3 are related to the findings of Vos (1977), referred to in the introduction, one might conclude that the interval-produced accent decreases with tone duration. Another argument against the energy-integration hypothesis could be the actual strength of the interval-produced accent.

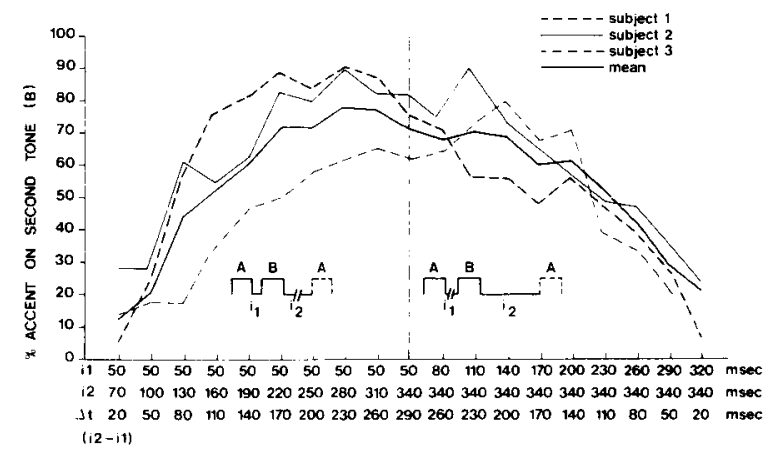

Figure 5. Percentage of perceived accent on last tone of group in sequences consisting of repeated groups of two tones, as a function of the duration of the Intervals 11 and 12 . Tone duration = 150 msec; durations of intervals are indicated in the figure. Inserts: one period of sequence. 


\section{EXPERIMENT 4}

Experiment 4 is an attempt to determine more directly the strength of the accents found in equitone sequences, and especially of the interval-produced accents. The method is similar to that used by Buytendijk and Meesters (1942). The strength of the accent on the second tone is measured by the amount that the intensity of the first tone of the group must be raised just to balance the accent on the second tone.

\section{Method}

Only one subject (Subject 1 from the preceding experiments) participated in Experiment 4. The data presented should, therefore, be considered as only an indication of the strength of the effect. The subject was presented with the stimuli of Experiment 1 and asked to adjust the intensity of the first tone, by turning a knob, until the two tones sounded equally accented. Every new stimulus sequence was presented with equal intensity of the two tones, namely $50 \mathrm{~dB}$ SPL. The stimuli were presented in a random order. Each stimulus was presented 24 times in a total of 12 sessions.

\section{Results and Discussion}

According to the subject, this task was easier to perform than that of the other experiments. Now, by turning the knob and subsequently adjusting the intensity such that the two tones appeared equally accented, the subject could move the accent from tone $A$ to tone B. Still, it took a number of practice trials before the subject started to generate consistent data. The main reason the task was not as easy as it would seem is that even when the two tones are correctly adjusted, that is, such that they seem to be equally accented, the sequence may still appear to be very "unrhythmical" as a result of the complex ratios of the tone-onset intervals (Povel, 1981).

The results are shown in Figure 6 together with the curve that the subject produced in Experiment 1. The data indicate, first, that the interval-produced

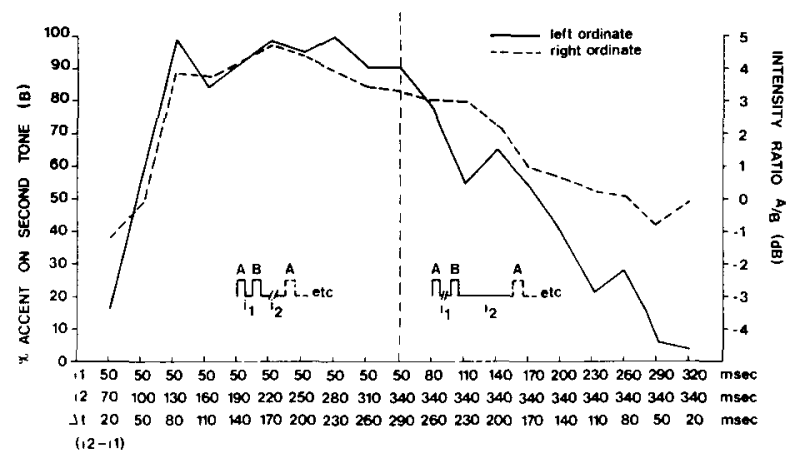

Figare 6. Dashed line shows adjusted intensity ratio of the tones at which they sound equally accented (right ordinate). Drawn line shows the curve the subject produced in Experiment 1 (left ordinate). accent is quite a robust phenomenon. An increase of about $4 \mathrm{~dB}$ in intensity is needed to balance it. The intensity difference limen for $1,000-\mathrm{Hz}$ tones at $50 \mathrm{~dB}$ is less than $.7 \mathrm{~dB}$ (Riesz, 1928). A tone with a 2-dB higher intensity than the surrounding tones will invariably be perceived as accented (Thomassen, Note 2). Second, the data indicate that the accent perceived on the first tone in the less differentiated sequences is not very strong, since it is easily compensated for.

\section{GENERAL DISCUSSION}

This study describes a determinant of accent in tone sequences not reported before. It was found that the relative length of a silent interval following a tone in equitone sequences is an important determiner of a perceived accent on that tone. The longer the silent interval, the better the chance that that tone will be perceived as bearing an accent. The effect is limited in two respects: (1) The shorter interval must be less than about 250 msec. (2) There must be a minimal difference between the two intervals, which depends on the absolute length of the shorter interval.

Experiments carried out to explore possible explanations of the effect have indicated that the intervalproduced accent is not caused by energy integration. Two arguments are relevant. First, the supposed difference in energy between the two tones is completely determined by the differences in the internal persistence of the two tones, and only in the tails of those persistences. On the basis of the energy integration hypothesis, one would expect the interval-produced accent to be a weak phenomenon that could be easily overruled. Experiments 3 and 4 show, however, that the effect is quite strong. The second argument is related to the finding that in traditional studies of temporal summation, energy integration is not reported beyond $150 \mathrm{msec}$. In Experiment 3, however, the interval-produced accent was found beyond 200 msec.

At this point, we would therefore prefer a more processing-oriented type of explanation (such as Massaro's, mentioned above), in which it is assumed that the measurement of the relative strength of a tone takes time and that the processing is interrupted when a new tone enters. If this explanation is correct, we must assume that interruption of the processing always leads to an underestimation of the strength attribute. This explanation perhaps cannot directly account for the finding, in Experiment 3, of slightly weaker effects with longer tone durations. The answer must presumably be sought in the moment at which the process of establishing the strength of a tone can reasonably start. When discussing Massaro's (1970) study in the introduction, we referred to the difference between the attributes of pitch and loud- 
ness (strength). While the processing of the pitch of a tone can start almost immediately upon onset, determining the loudness of a tone, being principally an integration process, can start only after a monitoring period. From what we know of temporal summation, $150 \mathrm{msec}$ would be a good guess for the duration of this period.

In order to show the possible practical relevance of the phenomenon, we would like to give a number of examples of music fragments in which the intervalproduced accent possibly plays a role. If we generalize the principle of interval-produced accent as described in this study, it holds that in groups of tones of different note values (intertone onset interval) the tone with the longer note value will be perceived as accented even if the actual tone durations are equal. If the tone with the longer note value is performed with a longer tone duration (which is possible but not necessary), there is of course even more reason for that tone to be perceived as being accented. In the examples given in Figure 7 , the locally determined interval-produced accents, indicated by the symbol 0 , are compatible with the accent structure of the meter at some places but not at others. Incompatibility arises when a tone with a relatively long note value, producing an interval-produced accent, occurs at a weak or unaccented beat. Every musician will recognize those instances (indicated in the figure by an asterisk) as creating a special rhythmical tension. Moreover, the performer will know that examples 2 and 3 are hard to perform. Those sequences are ambivalent because of the incompatibility of the local and structural accents. We have deliberately chosen examples without genuine syncopation (Cooper \& Meyer, 1971), in which the strong beat is not realized but displaced, because syncopation causes a strong rhythmical tension for its own sake.

Of course, the suggested role of the intervalproduced accent in the perception of the music given
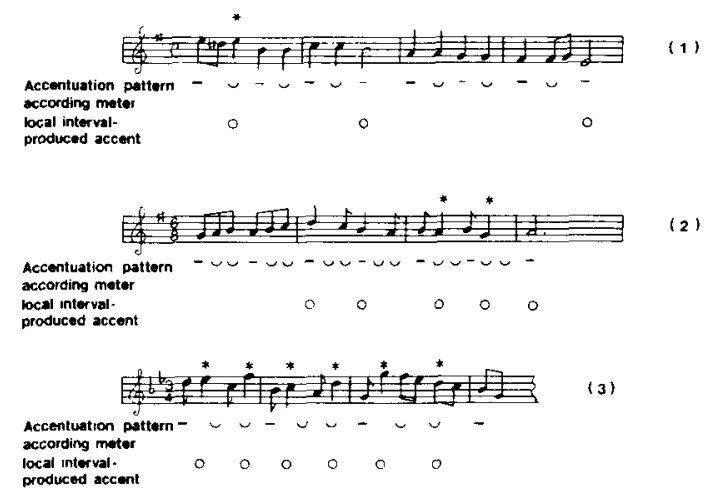

Figure 7. Music fragments showing interaction of accent structure from meter and locally determined Interval-produced accent. - Indicates incompatibillity (see text). (1) A. Goedike: Dance. (2) Anonymous. (3) $\mathrm{G}$. Ph. Telemann: Allegro from Fantasia in G Minor for fiute solo. as examples is completely speculative. Its actual meaning for the perception of rhythmical patterns can be determined only within a broader framework in which the roles and interactions of the different types of accents mentioned in the introduction, as well as of the "interval-produced" accent studied in this paper, are clearly defined. Such a framework has still to be developed; research toward reaching this goal is in progress.

\section{REFERENCE NOTES}

1. Thomassen, J. Melodic accent: Experiments and a tentative model (Tech. Rep. 361). Eindhoven: I.P.O., 1979.

2. Thomassen, J. Waarneming van geringe dynamische accentuering in toonreeksen (Tech. Rep. 999). Eindhoven: I.P.O., 1977.

\section{REFERENCES}

Boone, M. M. Loudness measurements on pure tone and broad band impulsive sounds. Acustica, 1973, 29, 198-204.

Buytendisk, F. J. J., \& Meesters, A. Duration and course of the auditory sensation. Commentationes Pontificia Academia Scientiarum, 1942, 6, 557-576.

COOPER, G., \& MEYER, L. B. The rhythmic structure of music. Chicago: University of Chicago Press, 1971.

Elliot, L. Backward and forward masking of probe tones of different frequencies. Journal of the Acoustical Society of America, 1962, 34, 1116-1117. (a)

ELlIOT, L. Backward masking: Monotic and dichotic conditions. Journal of the Acoustical Society of America, 1962, 34, 11081115. (b)

Elmasian, R., \& Galambos, R. Loudness enhancement: Monaural, binaural and dichotic. Journal of the Acoustical Society of America, 1975, 58, 229-234.

Fraisse, P. Les structures rythmiques. Louvain: Publications Universitaires de Louvain, 1956.

HARRELL, T. W. Factors affecting preference and memory for auditory rhythms. Journal of Genetic Psychology, 1937, 16, 427-469.

Koenler, W. Zur Theorie des Sukzessivvergleichs und der Zeitfehler. Psychologische Forschung, 1923, 4, 115-175.

Massaro, D. W. Preperceptual auditory images. Journal of Experimental Psychology, 1970, 85, 411-417.

Massaro, D. W. Preperceptual images, processing time and perceptual units in auditory perception. Psychological Review, $1972,79,124-145$.

NeEDHAM, J. G. The time-error as a function of continued experimentation. American Journal of Psychology, 1934, 46, 558-567.

Ne188Er, U. Cognitive psychology. New York: Appleton-CenturyCrofts, 1967.

Plomp, R. Rate of decay of auditory sensation. Journal of the Acoustical Society of America, 1964, 36, 277-282.

Postman, L. The time-error in auditory perception. American Journal of Psychology, 1946, 56, 193-219.

Povel, D. J. Internal representation of simple temporal patterns. Journal of Experimental Psychology: Human Perception and Performance, 1981, 7, 3-18.

ReichardT, W., \& Niese, $H$. Choice of sound duration and silent intervals for test and comparison signals in the subjective measurement of loudness level. Journal of the Acoustical Society of America, 1970, 47, 1083-1090.

RIEsz, R. R. Differential sensitivity of the ear for pure tones. Physical Review, 1928, 31, 867-875. 
Samollova, I. K. Masking of short tone signals as a function of the time interval between masked and masking sounds. Biofizika, $1959,4,555.558$ r

Vos, P. G. Temporal duration factors in the perception of auditory rhythmic patterns. Scientific Aesthetics, 1977, 1, 183-199.

Wilson, R. H., \& CARHART, R. Forward and backward masking: Interaction and additivity. Journal of the Acoustical Society of America, 1971, 49, 1254-1264.

Woodrow, H. A quantitative study of rhythm. In Archives of Psychology. No. 14. Columbia University Contributions to Philosophy and Psychology (Vol. 18, No. 1). New York: The Science Press, 1909.

ZwislockI, J. J. Temporal summation of loudness: An analysis. Journal of the Acoustical Society of America, 1969, 46, 431-441.
ZwisLocki, J. J. Masking: Experimental and theoretical aspects of simultaneous, forward, backward and central masking. In E. C. Carterette \& M. P. Friedman (Eds.), Handbook of perception (Vol. 4). New York: Academic Press, 1978.

Zwislocki, J. J., Ketkah, I., Cannon; M. W., \& Nodar, R. H. Loudness enhancement and summation in pairs of short sound bursts. Perception \& Psychophysics, 1974, 16, 91-95.

Zwislocki, J. J., \& Sokolich, W. G. On loudness enhancement of a tone burst by a preceding tone burst. Perception \& Psychophysics, 1974, 16, 87-90.

(Manuscript received May 26, 1981;

revision accepted for publication September 24, 1981.) 